DOI: $10.1002 / 201500214$

Article type: Full paper

\title{
Ferroelectric domain structures in low-strain $\mathrm{BaTiO}_{3}$
}

Arnoud S. Everhardt*, Sylvia Matzen, Neus Domingo, Gustau Catalan, Beatriz Noheda*

\author{
A. S. Everhardt, Dr. S. Matzen, Prof. B. Noheda \\ Zernike Institute for Advanced Materials \\ University of Groningen \\ 9747 AG Groningen, The Netherlands \\ E-mail: a.s.everhardt@rug.nl, b.noheda@rug.nl \\ Dr. N. Domingo, Prof. G. Catalan \\ Catalan Institute of Nanoscience and Nanotechnology (ICN2) \\ CSIC \\ Barcelona Institute of Science and Technology \\ Campus Universitat Autonoma de Barcelona, Bellaterra \\ 08193 Barcelona, Spain \\ Prof. G. Catalan \\ ICREA \\ 08193 Barcelona, Spain \\ Present address: Dr. S. Matzen \\ Institut d'Electronique Fondamentale, UMR CNRS \\ University Paris Sud \\ 91405 Orsay CEDEX, France
}

Keywords: ferroelectric, piezoelectric, $\mathrm{BaTiO}_{3}$ thin films, ferroelastic domains, pulsed laser deposition

Epitaxial strain in ferroelectric films offers the possibility to enhance the piezoelectric performance utilizing low crystal symmetries and high density of domain walls. Ferroelectric $\mathrm{BaTiO}_{3}$ has been predicted to order in a variety of phases and domain configurations when grown under low strain on low mismatched substrates, but little experimental evidence of that region of the phase diagram exist. In this work, epitaxial $\mathrm{BaTiO}_{3}$ thin films are grown on $\mathrm{NdScO}_{3}$ substrates under $\sim 0.1 \%$ strain. A monoclinic $\mathrm{ca}_{1} / \mathrm{ca}_{2}$ phase, with $90^{\circ}$ periodic inplane domain configuration and small additional out-of-plane component of polarization, has been stabilized at room temperature and investigated by Piezoelectric Force Microscopy and $\mathrm{X}$-ray Diffraction. Above $50{ }^{\circ} \mathrm{C}$ this phase is transformed into an a/c phase with alternating 


\section{WILEY-VCH}

in-plane and out-of-plane polarizations and forming zigzag domain walls between uppolarized and down polarized superdomains. Both types of domain patterns are highly anisotropic giving rise to very long domain walls. Above $130{ }^{\circ} \mathrm{C}$ the paraelectric phase is observed. The occurrence of a phase transition close to room temperature, a low symmetry $\mathrm{ca}_{1} / \mathrm{ca}_{2}$ phase and the formation of periodic domains make of this material a promising candidate for high piezoelectric response.

\section{Introduction}

Interplay between strain and polarization in ferroelectric perovskite epitaxial films can result in new polar states ${ }^{[1,2]}$ and new functionalities ${ }^{[3-8]}$. Strain relaxation by the formation of ferroelastic domains can allow further functionalization due to the presence of domain walls ${ }^{[9-12]}$ and periodic nanoscale structures ${ }^{[1,13-17]}$. The high piezoelectricity found in $\mathrm{PbZr}_{1-\mathrm{x}} \mathrm{Ti}_{\mathrm{x}} \mathrm{O}_{3}(\mathrm{PZT})$ and related $\mathrm{PbTiO}_{3}$ solid solutions materials originates in the existence of a monoclinic crystal structure and the formation of nanodomains at the Morphotrophic Phase Boundary (MPB) ${ }^{[18-20]}$. Ferroelastic nanodomain structures and low-symmetry monoclinic phases help accommodating the large elastic forces that develop at the MPB, providing elastic matching at the internal interfaces and inducing large piezoelectricity. In addition, the formation of ferroelastic nanodomains in thin films gives rise to novel effects such as flexoelectric polarization rotation in a/c domains ${ }^{[1]}$, enhanced piezoelectricity by the flexoelectric effect in PZT nanostructures ${ }^{[21]}$ or monoclinic areas of largely enhanced piezoresponse close to domain walls ${ }^{[22]}$. Thus, using epitaxial strain to induce different combinations of nanodomains with well-defined orientations and periodicities (domain engineering) opens a route to improved piezoelectrics, especially beneficial for the design of lead-free materials ${ }^{[23,24]}$. 


\section{WILEY-VCH}

In classical lead-free ferroelectric $\mathrm{BaTiO}_{3}$ single crystals, ferroelastic and ferroelectric $90^{\circ}$ domains and non-ferroelastic and ferroelectric $180^{\circ}$ domains are observed at room temperature ${ }^{[25-29]}$, consistent with the tetragonal crystal structure of the bulk crystal. Predictions for epitaxially strained $\mathrm{BaTiO}_{3}$ thin films have resulted in different crystal symmetries and, thus, different domain structures ${ }^{[30-37]}$. Particularly interesting are the predictions for films under low-strain values: even under zero nominal strain, the structure is modified by the elastic constrains imposed by the substrate and the different thermal expansion of film and substrate. Experimental realization of these phases is now becoming possible thanks to using substrates of the Rare-Earth Scandate family ${ }^{[38,39]}$. In particular, using a Landau-Ginsburg-Devonshire-type nonlinear phenomenological theory, Koukhar et al. reported the $\mathrm{BaTiO}_{3}$ phase diagram shown in Figure ${ }^{[30]}$. This and other related work ${ }^{[32,40,41]}$ have predicted a rich and flat energy landscape with large variety of single-, multiand metastable domain phases. Next to these many phase transitions (around which the piezoelectric responses are expected to greatly increase), monoclinic aa*/ca* and $\mathrm{ca}_{1} / \mathrm{ca}_{2}$ phases, not present in single crystals, have been predicted for low-strain values. . In this paper we test those predictions experimentally. We have stabilized two different domain structures with long and well-ordered domains: a room temperature monoclinic $\mathrm{ca}_{1} / \mathrm{ca}_{2}$ phase and either an a/c or an aa*/ca* phase above $50^{\circ} \mathrm{C}$.

\section{Results and discussion}

\section{1. $\mathrm{BaTiO}_{3}$ growth on $\mathrm{NdScO}_{3}$ substrate}

In this work $\mathrm{BaTiO}_{3}(001)_{p c}$ films have been grown on $\mathrm{NdScO}_{3}(001)_{p c} /(110)_{o}$ substrates (where subscripts $p c$ and $o$ refer to the pseudocubic and orthorhombic unit cells respectively) as $\mathrm{NdScO}_{3}$ is the only currently commercial available substrate which provides low strain: the $\mathrm{NdScO}_{3}$ in-plane lattice parameters are $4.002 \AA(100)_{p d} /(002)_{o}$ and $4.014 \AA(010)_{p c} /(-110)_{o}{ }^{[38]}$ 


\section{WILEY-VCH}

which produce nominal room temperature misfit strains $\varepsilon_{\mathrm{s}}$ of $-0.1 \%$ compressive strain and $+0.2 \%$ tensile strain, respectively, for $\mathrm{BaTiO}_{3}{ }^{[42]}$; the average mismatch strain is therefore only $+0.05 \%$, i.e. marginally tensile but very close to zero.

$\mathrm{BaTiO}_{3}$ films with thickness of $90 \mathrm{~nm}$ were grown on a $\mathrm{NdScO}_{3}$ substrate (miscut angle 0.05$0.2^{\circ}$ ), with a $6 \mathrm{~nm}$ thick, fully-strained, metallic $\mathrm{SrRuO}_{3}$ bottom electrode, using Pulsed Laser Deposition (PLD). Atomic Force Microscopy (AFM) images of the substrate (and the electrode, not shown here) show flat terraces (see Figure 2a ), while the surface of $\mathrm{BaTiO}_{3}$ reveals an island growth-like structure (Figures $\mathbf{2 b - c )}$ ) with 4-5 clearly distinguishable unit cell steps. $\mathrm{NdScO}_{3}, \mathrm{SrRuO}_{3}$ and $\mathrm{BaTiO}_{3}$ peaks are present in the $\theta-2 \theta$ X-Ray Diffraction (XRD) scan around the $(001)_{p c}$ Bragg peak ${ }^{[43-48]}$ (Figure 2d) with superimposed finite size oscillations for the $\mathrm{SrRuO}_{3}$ and $\mathrm{BaTiO}_{3}$ films, indicating the high quality of the interfaces. Figure 2e and Figure 2f show the off-specular $\mathrm{BaTiO}_{3}(204)_{p c}$ and $\mathrm{NdScO}_{3}(620)_{o}$ Bragg peaks of films grown with 0.20 mbar and 0.15 mbar oxygen pressure $\left(\mathrm{P}_{\mathrm{O} 2}\right)$ respectively. For $\mathrm{P}_{\mathrm{O} 2}=0.20$ mbar, the in-plane lattice parameters are equal for $\mathrm{BaTiO}_{3}$ and $\mathrm{NdScO}_{3}$, indicating full epitaxy. Instead, the films grown with $\mathrm{P}_{\mathrm{O} 2}=0.15$ mbar have additional peaks in the inplane direction, caused by periodic domains. Indeed, changing $\mathrm{P}_{\mathrm{O} 2}$ during growth seems to affect the domain ordering: films grown at $\mathrm{P}_{\mathrm{O} 2}=0.15$ mbar films have ordered periodic domains with $100 \mathrm{~nm}$ periodicity, films grown at $\mathrm{P}_{\mathrm{O} 2}=0.18$ mbar show the same domain periodicity but with increased disorder and films grown at $\mathrm{P}_{\mathrm{O} 2}=0.20$ mbar films have disordered, non-periodic domains with sizes of about $500 \mathrm{~nm}$. One way to explain these different results between these two kind is by considering that a higher concentration of oxygen vacancies $\left(\mathrm{V}_{\mathrm{O}}\right)$ allows the lattice to better accommodate the strain by lowering the nucleation energy of domain walls, therefore promoting strain relaxation by an increasing amount of elastically favourable domains. Moreover, contrary to the usual situation where $\mathrm{V}_{\mathrm{O}}$ increases the lattice parameters ${ }^{[49]}$, it is found that increasing $\mathrm{P}_{\mathrm{O} 2}$ (or decreasing $\mathrm{V}_{\mathrm{O}}$ ) in the range from 0.15 to 0.20 mbar gives rise to an increase of the out-of-plane lattice parameter 


\section{WILEY-VCH}

from 4.015 to $4.022 \pm 0.002 \AA$. An increase is also found when the domain pattern for a fixed $\mathrm{V}_{\mathrm{O}}$ changes (by temperature) from a mostly in-plane $\mathrm{ca}_{1} / \mathrm{ca}_{2}$ to a more out-of-plane $\mathrm{a} / \mathrm{c}$ domains. This increase of lattice parameter for lower $\mathrm{V}_{\mathrm{O}}$ can be explained by considering an increase of out-of-plane domains when the film domain pattern gets more disordered.

\subsection{Piezoelectric Force Microscopy domain configurations}

Temperature-dependent Piezoelectric Force Microscopy (PFM) was used to characterize the domain states. Dual AC Resonance Tracking (DART) mode was used to separate the piezoresponse signal from the strong topological features from the island-like surface. $90^{\circ}$ inplane domains with walls along the $\langle 110\rangle_{p c}$ axes are observed from lateral PFM images at room temperature, as seen in Figure 3a-b. These are consistent with both a periodic $\mathrm{a}_{1} / \mathrm{a}_{2}$ or $\mathrm{ca}_{1} / \mathrm{ca}_{2}$ domain structure (see Figure $\mathbf{S 1}$ for more details) with a domain period of $100 \mathrm{~nm}$ and a strong preferential orientation for the walls to be parallel to one of the $\langle 110\rangle_{p c}$ axes. The domains with the polarization along $\langle 010\rangle_{p c}$ are about two times larger than the domains with polarization along $\langle 100\rangle_{p c}$, caused by the larger substrate lattice parameter along the $[010]_{p c}$ direction.

At $50{ }^{\circ} \mathrm{C}$, a phase transition to an a/c phase takes place, as shown in Figure 3c-d. The transition is accompanied by a decrease of the domain periodicity to $70 \mathrm{~nm}$. All the domain walls are then rotated by $45^{\circ}$ and become parallel to only the $(010)_{p c}$ plane and not the $(100)_{p c}$ plane, because the significantly larger substrate in-plane lattice parameter towards $[010]_{p c}$ makes it favorable to have the long axis of the a-domains (the polarization axis) only along that direction. These domains are exceptionally long: domains of at least $10 \mu \mathrm{m}$ without disturbances (Figure 3e) are seen and seem to run across the full sample dimensions (5 $\mathrm{mm})$. These domains are gathered in superdomains that differ from each other by a $180^{\circ}$ rotation of the polarization (see Figure S2 for more details). A zig-zag superdomain wall is formed in 


\section{WILEY-VCH}

Figure 3f-g, similar to those reported by Feigl et al. ${ }^{[50]}$ in PZT films. The zigzag walls allow both in-plane and out-of-plane polarization in the a/c domains to change by a phase shift of the in-plane and out-of-plane components at both sides of the wall.

The minimum expected domain period of a/c phases can be calculated by considering the bulk c/a ratio and the substrate lattice parameter ${ }^{[15]}$. Following the calculations for this material combination, a $35 \mathrm{~nm}$ domain size for both a- and c-domains is obtained, corresponding exactly to the observed $70 \mathrm{~nm}$ total domain periodicity.

The resolution in these PFM images, however, is not enough to assign observed out-of-plane signal (not shown) in the apparent $\mathrm{a}_{1} / \mathrm{a}_{2}$ phase to real polarization (thus corresponding to the monoclinic $\mathrm{ca}_{1} / \mathrm{ca}_{2}$ phase) or crosstalk from in-plane polarization ${ }^{[29]}$; neither can observed additional in-plane polarization in the a/c phase be conclusively assigned to the monoclinic aa*/ca* phase or imperfect alignment of the sample. X-ray diffraction is used to conclude about the nature of the domain configurations.

\subsection{X-ray Diffraction domain configurations}

In-plane and out-of-plane XRD Reciprocal Space Maps (RSM) of the $(100)_{p c},(110)_{p c}$ and $(001)_{p c}$ Bragg peaks in the room temperature phase, as shown in Figure 4a-c, display additional modulations in the diffuse scattering, corresponding to domain periodicity. It can be seen that the domains run along the $\langle 110\rangle_{p c}$ direction with a real-space periodicity of 100 $\mathrm{nm}^{[51]}$, fully consistent with the PFM data. Satellites around the different Bragg peaks do not present two-fold symmetry, as expected for a high preferential orientation of the domain walls, also observed in PFM. These in-plane XRD maps are similar to those observed for $a_{1} / a_{2}$ domain structures in $(\mathrm{Pb}, \mathrm{Sr}) \mathrm{TiO}_{3}{ }^{[13]}$. However, additional intensity modulations (with the same $100 \mathrm{~nm}$ real-space in-plane periodicity) can also be seen around the out-of-plane $(001)_{p c}$ Bragg peak (see Figure 4c). Since XRD intensity scales as $\left|e^{i k_{z} z}\right|^{2}$, a modulation of the z- 


\section{WILEY-VCH}

component of the atomic displacemts (and thus, out-of-plane polarization) is required. All the evidence combined seem to point to the predicted $\mathrm{ca}_{1} / \mathrm{ca}_{2}{ }^{[30]}$ phase, with a small out-of-plane polarization instability. One can notice that a projection of the domain periodicity (running along the $[110]_{p c}$ direction) onto other in-plane axes of the RSM gives a continuous apparent different domain period, calculated by apparent domain size $=$ domain period $/ \cos ($ angle of rotation away from $[110]_{p c}$ ). So along the $\langle 100\rangle_{p c}$ directions the apparent domain length is $140 \mathrm{~nm}$ and along the $[1-10]_{p c}$ direction (parallel to the walls) the domain length is infinite, leading to the disappearance of the domain peaks. The images can be found in Figure S3. Increasing the temperature to $80^{\circ} \mathrm{C}$, an a/c phase is observed. The XRD RSMs around the $(010)_{p c}$ and $(100)_{p c}$ Bragg peaks are given in Figure 4d-e. Intensity modulations are observed in the diffuse scattering in the $[010]_{p c}$ direction around the $(010)_{p c}$ Bragg peak with a realspace periodicity of $70 \mathrm{~nm}$; while no periodic features are observed around the $(100)_{p c}$ Bragg peak. This is different than the observation of a/c domain structures in ultrathin $\mathrm{PbTiO}_{3}$ grown on $\mathrm{DyScO}_{3}{ }^{[52]}$, which produced periodic modulations in the $[100]_{p c}$ direction and domain peaks around the $(100)_{p c}$ Bragg peak. The difference in this work can be explained by the anisotropy of the walls in the present case (no domain wall along $(100)_{p c}$ ). XRD RSM around the $(001)_{p c}$ Bragg peak with a $70 \mathrm{~nm}$ real-space in-plane periodicity is given in Figure $4 \mathbf{f}$. The intensity of these out-of-plane domain peaks is one order of magnitude higher than of the corresponding domain peaks for $\mathrm{a} \mathrm{ca}_{1} / \mathrm{ca}_{2}$ phase, showing one order of magnitude higher outof-plane polarization modulation in this a/c phase.

The theoretical phase diagram (see Figure 1) predicts a phase transition from the $\mathrm{ca}_{1} / \mathrm{ca}_{2}$ phase to the aa*/ca* phase, and a subsequent transition to the a/c phase for increasing temperature.

Using the current methods we are unable to distinguish the a/c from the aa*/ca* phase, so it is well possible that we are observing a monoclinic aa*/ca* phase instead of the a/c phase; there might be an additional phase transition between these last two phases. 


\section{WILEY-VCH}

For both structures, the periodic modulations of the diffuse scattering around the $(001)_{p c}$ Bragg peaks are detected at reciprocal lattice points coinciding with the a- and c-lattice parameters of bulk $\mathrm{BaTiO}_{3}$. These bulk out-of-plane lattice parameters are conserved upon azimuthal rotation around $[001]_{p c}$, while the in-plane positions of the periodic modulations are changed, reflecting a change in periodicity. This observation suggests a lattice relaxation of the film from fully strained (at the Bragg peak) to fully relaxed a- and c-lattices. A strain gradient is thus most likely produced in both phases, inducing a flexoelectric polarization and thus an additional lowering of the symmetry, similar to what is observed in $\mathrm{PbTiO}_{3} \mathrm{a} / \mathrm{c}$ domains ${ }^{[1]}$.

\subsection{Phase coexistence}

If the material is cooled down fast enough, metastable a/c domains are observed to coexist within the $\mathrm{ca}_{1} / \mathrm{ca}_{2}$ phase at room temperature (Figure S4) for several days, giving evidence for the first order character of the $\mathrm{a} / \mathrm{c}$ to $\mathrm{ca}_{1} / \mathrm{ca}_{2}$ phase transition. This observation stands in contrast to the theoretical prediction that ferroelectric transitions in mono-domain epitaxial $\mathrm{BaTiO}_{3}$ should be second order due to clamping-induced renormalization of the quadratic coefficient of the polarization in the free energy ${ }^{[33]}$, but it is fully consistent with the prediction for the ferroelectric-ferroelectric transition in polydomain films with a small amount of tensile strain ${ }^{[30]}$. Single crystal $\mathrm{BaTiO}_{3}$ has similar remanence with monoclinic phase stability up to $100 \mathrm{~K}$ away from a thermotropic phase boundary ${ }^{[22,53]}$.

\subsection{Ferroelectric-to-paraelectric phase transition}

The paraelectric transition temperature, the Curie temperature $\left(\mathrm{T}_{\mathrm{C}}\right)$, of $130{ }^{\circ} \mathrm{C}$ is just slightly above the bulk value, confirming the low-strain state of the film as a high strain would 


\section{WILEY-VCH}

increase $\mathrm{T}_{\mathrm{C}}$ further ${ }^{[3]}$. Local piezoelectric loops can be obtained in the two ferroelectric states $\left(\mathrm{ca}_{1} / \mathrm{ca}_{2}\right.$ and a/c phases) as shown in Figure 5a. Above $\mathrm{T}_{\mathrm{C}}$ the piezoelectric loops disappear (Figure 5b), proving the ferroelectric origin of the piezoelectric loop and, simultaneously, the phase transition ${ }^{[54]}$. Furthermore, the strained out-of-plane lattice parameter (in Figure 5c) decreases gradually near $\mathrm{T}_{\mathrm{C}}$ (and at the $50{ }^{\circ} \mathrm{C}$ phase transition a small increase can be seen). Since the lattice parameter scales with $\mathrm{P}^{2}$ (P is polarization) ${ }^{[55]}$, it represents a gradual decrease of the order parameter and thus a second order phase transition, representing a ferroelectric thin film under strain.

\section{Conclusion}

Two phase transitions have been stabilized in low-strain $\mathrm{BaTiO}_{3}$ thin films on $\mathrm{NdScO}_{3}$ substrates: the $\mathrm{ca}_{1} / \mathrm{ca}_{2}$ phase at room temperature changes into an $\mathrm{a} / \mathrm{c}$-like phase $(\mathrm{a} / \mathrm{c}$ or aa*/ca* phase) above $50{ }^{\circ} \mathrm{C}$, with the Curie temperature $\mathrm{T}_{\mathrm{C}}$ of the paraelectric phase transition at $130{ }^{\circ} \mathrm{C}$. The polarization freedom in this material is very large, as indicated by the monoclinic room temperature $\mathrm{ca}_{1} / \mathrm{ca}_{2}$ phase, the probable additional flexoelectric polarization induced by the nanodomains, the presence of metastable phases and phase coexistence and finally by the fact that there is a phase transition close to room temperature. So low-strain $\mathrm{BaTiO}_{3}$ thin films show large potential for high piezoelectric responses. Additionally, contrary to most materials with such polarization freedom, $\mathrm{BaTiO}_{3}$ on $\mathrm{NdScO}_{3}$ exhibits wellordered structures, especially the exceptionally ordered millimeter long a/c domains. This high order is critical in designing devices where ordered structures can have strong contributions and be exploited for tailoring physical properties.

\section{Experimental Section}




\section{WILEY-VCH}

$\mathrm{BaTiO}_{3}$ thin films have been deposited by Pulsed Laser Deposition, using a Lambda Physik COMPex Pro $205 \mathrm{KrF}(\lambda=248 \mathrm{~nm})$ excimer ultraviolet laser and a Twente Solid State Technology reflection high-energy electron diffraction (RHEED) vacuum system. The target was a $\mathrm{BaTiO}_{3}$ single crystal $(\mathrm{MaTecK} \mathrm{GmbH})$ and the deposition parameters were a background pressure of $10^{-7}$ mbar, a substrate temperature of $700-800{ }^{\circ} \mathrm{C}$, a laser energy of 2 $\mathrm{Jcm}^{-2}$, a spotsize of $2.6 \mathrm{~mm}^{2}$, a target-substrate distance of $50 \mathrm{~mm}$, a repetition rate of $5 \mathrm{~Hz}$, cooling rate of $5{ }^{\circ} \mathrm{C} / \mathrm{min}$ at 300 mbar $\mathrm{P}_{\mathrm{O} 2}$ after the growth and the growth $\mathrm{P}_{\mathrm{O} 2}$ was varied between 0.15 and 0.20 mbar. $\mathrm{NdScO}_{3}$ substrates (CrysTec $\mathrm{GmbH}$ ) were pre-treated to obtain single termination (adapted from Ref. ${ }^{[56]}$ ) for 4 hours at $900{ }^{\circ} \mathrm{C}$ in an oxygen flow and subsequently with $12 \mathrm{M} \mathrm{NaOH}$ and $1 \mathrm{M} \mathrm{NaOH}$ for several hours and a water/ethanol cleaning step.

Characterization was performed using AFM (Veeco Dimension V) and PFM in Dual AC Resonance Tracking (DART) mode (Asylum). X-ray diffraction measurements were done at both the PETRA III P08 beamline (DESY, Hamburg) operated at $9 \mathrm{keV}(\lambda=1.37761 \AA)$ and a Panalytical X'Pert MRD Cradle (four axes) laboratory diffractometer, copper X-ray generator tube $(\lambda=1.540598 \AA)$, both equipped with an Anton Paar heating stage.

\section{Supporting Information}

Supporting Information is available from the Wiley Online Library or from the author.

\section{Acknowledgements}

We are grateful to J. Schwarzkopf, J. Hlinka, T. Denneulin, E. Snoeck and C. Magen for useful discussions. Parts of this research were carried out at the light source Petra III at DESY, a member of the Helmholtz Association (HGF). We would like to thank F. Bertram and U. Rütt for assistance in using beamline P08. We are grateful to the alumni organization of the University of Groningen, De Aduarderking (Ubbo Emmius Fonds), and to the Zernike Institute for Advanced Materials for financial support.

Received: ((will be filled in by the editorial staff))

Revised: ((will be filled in by the editorial staff)) Published online: ((will be filled in by the editorial staff))

[1] G. Catalan, A. Lubk, A. H. G. Vlooswijk, E. Snoeck, C. Magen, A. Janssens, G. Rispens, G. Rijnders, D. H. A. Blank, B. Noheda, Nat. Mater. 2011, 10, 963. 


\section{WILEY-VCH}

[2] J. C. Yang, Q. He, S. J. Suresha, C. Y. Kuo, C. Y. Peng, R. C. Haislmaier, M. A. Motyka, G. Sheng, C. Adamo, H. J. Lin, Z. Hu, L. Chang, L. H. Tjeng, E. Arenholz, N. J. Podraza, M. Bernhagen, R. Uecker, D. G. Schlom, V. Gopalan, L. Q. Chen, C. T. Chen, R. Ramesh, Y. H. Chu, Phys. Rev. Lett. 2012, 109, 247606.

[3] K. J. Choi, M. Biegalski, Y. L. Li, A. Sharan, J. Schubert, R. Uecker, P. Reiche, Y. B. Chen, X. Q. Pan, V. Gopalan, L.-Q. Chen, D. G. Schlom, C. B. Eom, Science 2004, 306,1005 .

[4] I. B. Misirlioglu, S. P. Alpay, F. He, B. O. Wells, J. Appl. Phys. 2006, 99, 104103.

[5] J. H. Haeni, P. Irvin, W. Chang, R. Uecker, P. Reiche, Y. L. Li, S. Choudhury, W. Tian, M. E. Hawley, B. Craigo, A. K. Tagantsev, X. Q. Pan, S. K. Streiffer, L. Q. Chen, S. W. Kirchoefer, J. Levy, D. G. Schlom, Nature 2004, 430, 758.

[6] A. R. Damodaran, E. Breckenfeld, Z. Chen, S. Lee, L. W. Martin, Adv. Mater. 2014, 26,6341 .

[7] B. Cai, J. Schwarzkopf, E. Hollmann, M. Schmidbauer, M. O. Abdel-Hamed, R. Wördenweber, J. Appl. Phys. 2014, 115, 224103.

[8] E. Chernova, O. Pacherova, D. Chvostova, a. Dejneka, T. Kocourek, M. Jelinek, M. Tyunina, Appl. Phys. Lett. 2015, 106, 192903.

[9] S. Farokhipoor, B. Noheda, Phys. Rev. Lett. 2011, 107, 127601.

[10] T. Sluka, A. K. Tagantsev, D. Damjanovic, M. Gureev, N. Setter, Nat. Commun. 2012, 3,748 .

[11] T. Sluka, A. K. Tagantsev, P. Bednyakov, N. Setter, Nat. Commun. 2013, 4, 1808.

[12] G. Catalan, J. Seidel, R. Ramesh, J. Scott, Rev. Mod. Phys. 2012, 84, 119.

[13] S. Matzen, O. Nesterov, G. Rispens, J. A. Heuver, M. Biegalski, H. M. Christen, B. Noheda, Nat. Commun. 2014, 5, 4415.

[14] N. Balke, S. Choudhury, S. Jesse, M. Huijben, Y. H. Chu, A. P. Baddorf, L. Q. Chen, R. Ramesh, S. V. Kalinin, Nat. Nanotechnol. 2009, 4, 868.

[15] A. H. G. Vlooswijk, B. Noheda, G. Catalan, A. Janssens, B. Barcones, G. Rijnders, D. H. A. Blank, S. Venkatesan, B. Kooi, J. T. M. de Hosson, Appl. Phys. Lett. 2007, 91, 112901.

[16] L. Feigl, L. J. McGilly, C. S. Sandu, N. Setter, Appl. Phys. Lett. 2014, 104, 172904.

[17] V. Nagarajan, I. G. Jenkins, S. P. Alpay, H. Li, S. Aggarwal, L. Salamanca-Riba, a. L. Roytburd, R. Ramesh, J. Appl. Phys. 1999, 86, 595.

[18] B. Noheda, J. A. Gonzalo, L. E. Cross, R. Guo, S.-E. Park, D. E. Cox, G. Shirane, Phys. Rev. B 2000, 61, 8687. 


\section{WILEY-VCH}

[19] L. Bellaiche, A. García, D. Vanderbilt, Phys. Rev. Lett. 2000, 84, 5427.

[20] Y. M. Jin, Y. U. Wang, A. G. Khachaturyan, J. F. Li, D. Viehland, Phys. Rev. Lett. 2003, 91, 197601.

[21] M. S. Majdoub, P. Sharma, T. Çağin, Phys. Rev. B 2008, 78, 121407.

[22] T. T. A. Lummen, Y. Gu, J. Wang, S. Lei, F. Xue, A. Kumar, A. T. Barnes, E. Barnes, S. Denev, A. Belianinov, M. Holt, A. N. Morozovska, S. V. Kalinin, L.-Q. Chen, V. Gopalan, Nat. Commun. 2014, 5, 3172.

[23] S. Zhang, R. Xia, T. R. Shrout, J. Electroceram. 2007, 19, 251.

[24] J. Rödel, W. Jo, K. T. P. Seifert, E.-M. Anton, T. Granzow, D. Damjanovic, J. Am. Ceram. Soc. 2009, 92, 1153.

[25] H. Blattner, W. Känzig, W. Merz, Helv. Phys. Acta 1948, 22, 35.

[26] W. J. Merz, Phys. Rev. 1954, 95, 690.

[27] J. A. Hooton, W. J. Merz, Phys. Rev. 1955, 98, 409.

[28] A. Schilling, T. B. Adams, R. Bowman, J. Gregg, G. Catalan, J. Scott, Phys. Rev. B 2006, 74,1 .

[29] L. J. McGilly, A. Schilling, J. M. Gregg, Nano Lett. 2010, 10, 4200.

[30] V. G. Koukhar, N. A. Pertsev, R. Waser, Phys. Rev. B 2001, 64, 214103.

[31] Y. L. Li, L. Q. Chen, Appl. Phys. Lett. 2006, 88, 072905.

[32] O. Diéguez, S. Tinte, A. Antons, C. Bungaro, J. B. Neaton, K. M. Rabe, D. Vanderbilt, Phys. Rev. B 2004, 69, 212101.

[33] N. A. Pertsev, A. G. Zembilgotov, A. K. Tagantsev, Phys. Rev. Lett. 1998, 80, 1988.

[34] V. B. Shirokov, Y. I. Yuzyuk, B. Dkhil, V. V. Lemanov, Phys. Solid State 2008, 50, 928.

[35] G. Sheng, J. X. Zhang, Y. L. Li, S. Choudhury, Q. X. Jia, Z. K. Liu, L. Q. Chen, Appl. Phys. Lett. 2008, 93, 232904.

[36] V. B. Shirokov, Y. I. Yuzyuk, B. Dkhil, V. V. Lemanov, Phys. Rev. B 2007, 75, 224116.

[37] M. Marathe, C. Ederer, Appl. Phys. Lett. 2014, 104, 212902.

[38] R. Uecker, B. Velickov, D. Klimm, R. Bertram, M. Bernhagen, M. Rabe, M. Albrecht, R. Fornari, D. G. Schlom, J. Cryst. Growth 2008, 310, 2649. 


\section{WILEY-VCH}

[39] D. G. Schlom, L.-Q. Chen, C.-B. Eom, K. M. Rabe, S. K. Streiffer, J.-M. Triscone, Annu. Rev. Mater. Res. 2007, 37, 589.

[40] N. A. Pertsev, V. G. Koukhar, Phys. Rev. Lett. 2000, 84, 3722.

[41] Z. Gui, S. Prosandeev, L. Bellaiche, Phys. Rev. B 2011, 84, 214112.

[42] Strain is defined by $\varepsilon_{\mathrm{s}}=\left(\mathrm{a}_{\mathrm{sub}}-\mathrm{a}_{0}\right) / \mathrm{a}_{0}$, where $\mathrm{a}_{\text {sub }}$ is the substrate lattice parameter and $\mathrm{a}_{0}$ is the lattice parameter of freestanding cubic $\mathrm{BaTiO}_{3}$.

[43] C. Thompson, C. M. Foster, J. A. Eastman, G. B. Stephenson, Appl. Phys. Lett. 1997, $71,3516$.

[44] B. L. Henke, E. M. Gullikson, J. C. Davis, At. Data Nucl. Data Tables 1993, 54, 181.

[45] D. Waasmaier, A. Kirfel, Acta Crystallogr., Sect. A: Found. Adv. 1995, 51, 416.

[46] R. P. Liferovich, R. H. Mitchell, J. Solid State Chem. 2004, 177, 2188.

[47] K. J. Choi, S. H. Baek, H. W. Jang, L. J. Belenky, M. Lyubchenko, C.-B. Eom, Adv. Mater. 2010, 22, 759.

[48] G. H. Kwei, A. C. Lawson, S. J. L. Billinge, S. W. Cheong, J. Phys. Chem. 1993, 97, 2368.

[49] Y.-M. Kim, J. He, M. D. Biegalski, H. Ambaye, V. Lauter, H. M. Christen, S. T. Pantelides, S. J. Pennycook, S. V. Kalinin, A. Y. Borisevich, Nat. Mater. 2012, 11, 888.

[50] L. Feigl, L. J. McGilly, N. Setter, Ferroelectrics 2014, 465, 36.

[51] The real-space domain period can be calculated by using the in-plane distance between the Bragg peak and the domain peak in r.l.u., and then use the formula: real-space period $=$ substrace lattice parameter $/$ distance in r.l.u.

[52] G. Catalan, A. Janssens, G. Rispens, S. Csiszar, O. Seeck, G. Rijnders, D. H. A. Blank, B. Noheda, Phys. Rev. Lett. 2006, 96, 127602.

[53] Y. Gu, F. Xue, S. Lei, T. T. A. Lummen, J. Wang, V. Gopalan, L.-Q. Chen, Phys. Rev. B 2014, 90, 024104.

[54] C. W. Bark, P. Sharma, Y. Wang, S. H. Baek, S. Lee, S. Ryu, C. M. Folkman, T. R. Paudel, A. Kumar, S. V. Kalinin, A. Sokolov, E. Y. Tsymbal, M. S. Rzchowski, A. Gruverman, C. B. Eom, Nano Lett. 2012, 12, 1765.

[55] R. A. Cowley, Adv. Phys. 1980, 29, 1.

[56] J. E. Kleibeuker, G. Koster, W. Siemons, D. Dubbink, B. Kuiper, J. L. Blok, C.-H. Yang, J. Ravichandran, R. Ramesh, J. E. ten Elshof, D. H. A. Blank, G. Rijnders, $A d v$. Funct. Mater. 2010, 20, 3490. 

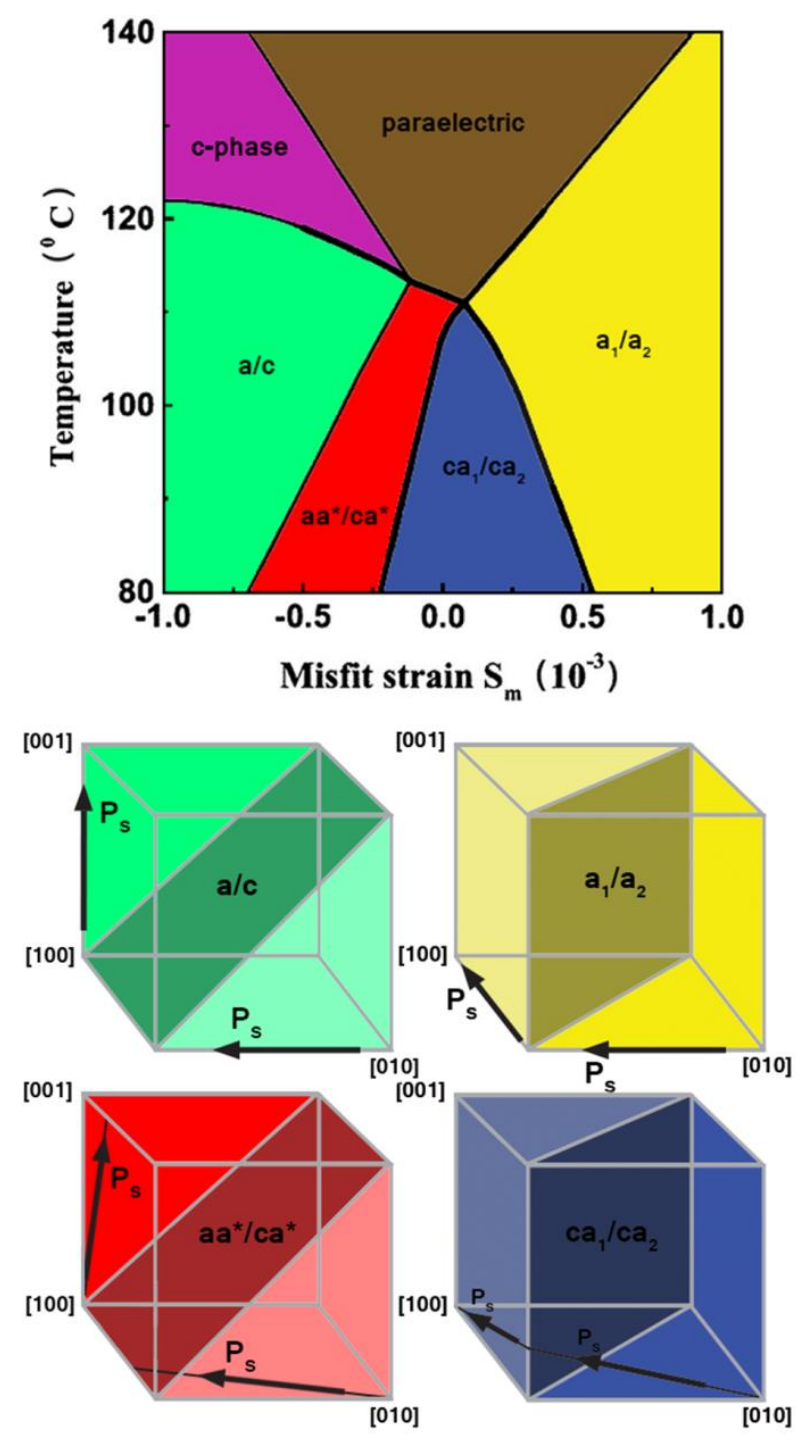

Figure 1. Pertsev phase diagram of misfit strain versus temperature for low-strain $\mathrm{BaTiO}_{3}$. Thick lines represent first order and thin lines represent second order phase transitions. The predicted phases are the paraelectric phase $\left(\mathrm{P}_{\mathrm{x}}=\mathrm{P}_{\mathrm{y}}=\mathrm{P}_{\mathrm{z}}=0\right)$, c-phase $\left(\mathrm{P}_{\mathrm{x}}=\mathrm{P}_{\mathrm{y}}=0, \mathrm{P}_{\mathrm{z}} \neq 0\right)$, a/c-phase $\left(P_{x} \neq 0, P_{y}=P_{z}=0\right.$ and $\left.P_{x}=P_{y}=0, P_{z} \neq 0\right)$, aa*/ca*-phase $\left(P_{x} \neq 0, P_{y} \neq 0, P_{z}=0\right.$ and $\left.\mathrm{P}_{\mathrm{x}}=0, \mathrm{P}_{\mathrm{y}} \neq 0, \mathrm{P}_{\mathrm{z}} \neq 0\right), \mathrm{a}_{1} / \mathrm{a}_{2}$-phase $\left(\mathrm{P}_{\mathrm{x}} \neq 0, \mathrm{P}_{\mathrm{y}}=\mathrm{P}_{\mathrm{z}}=0\right.$ and $\left.\mathrm{P}_{\mathrm{x}}=\mathrm{P}_{\mathrm{z}}=0, \mathrm{P}_{\mathrm{y}} \neq 0\right)$ and $c a_{1} / c_{2}$-phase $\left(P_{x} \neq 0, P_{y}=0, P_{z} \neq 0\right.$ and $\left.P_{x}=0, P_{y} \neq 0, P_{z} \neq 0\right)$.

Phase diagram adapted with permission from Ref. ${ }^{[30]}$ Copyright 2001, American Physical Society. 


\section{WILEY-VCH}

(a)

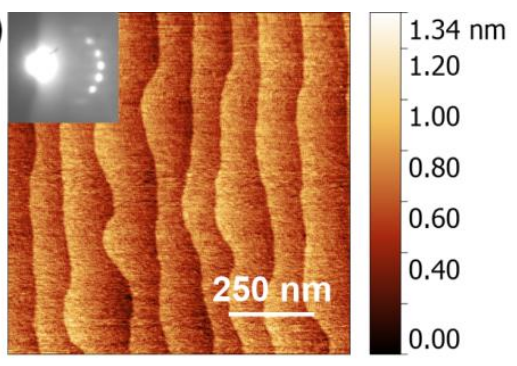

(d)

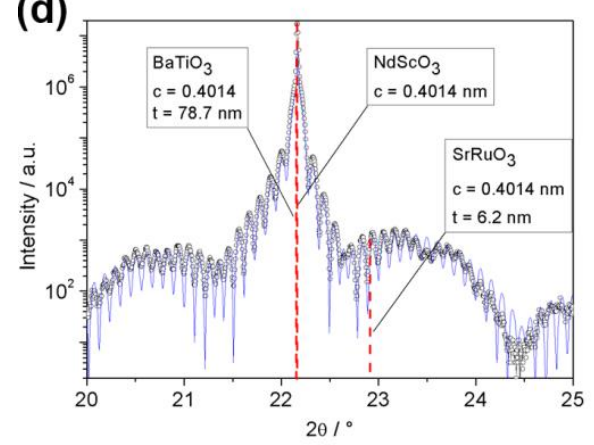

(b)

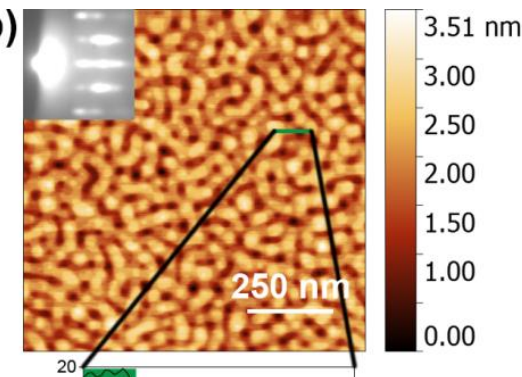

(c)

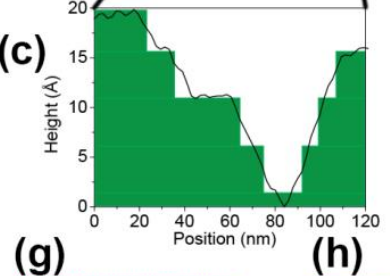

(e)

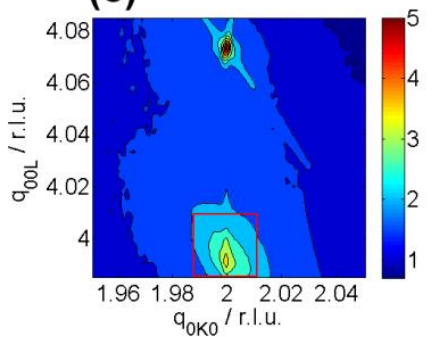

(f)

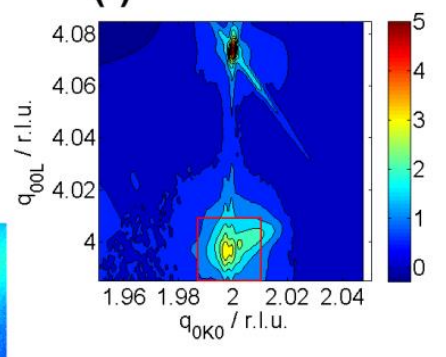

Figure 2. Epitaxial $\mathrm{BaTiO}_{3}$ film. a-b) AFM images of respectively $\mathrm{NdScO}_{3}$ substrate and $\mathrm{BaTiO}_{3}$ film. Insets: the Reflective High-Energy Electron Diffraction (RHEED) images show respectively the flat orthorhombic surface structure of $\mathrm{NdScO}_{3}$ and the island-growth mode of $\mathrm{BaTiO}_{3}$. c) Cross-section (data as black line, sketch of unit-cell steps in green) of the $\mathrm{BaTiO}_{3}$ islands displaying 4-5 unit cell steps, maintaining high quality epitaxy. d) $\theta / 2 \theta$ XRD scan (black circles) around (001) Bragg peak (film grown at $\mathrm{P}_{\mathrm{O} 2}=0.15 \mathrm{mbar}$ ). Fit of the data (blue line) with the fit parameters ( $\mathrm{c}$ is the c-axis lattice parameter and $\mathrm{t}$ is the thickness of the film) ${ }^{[43-48]}$. e-f) Reciprocal Space Maps around (204) $)_{p c}$ of both $\mathrm{BaTiO}_{3}$ film and $\mathrm{NdScO}_{3}$ substrate (corresponding to the (602) $)_{o}$ Bragg reflection of the substrate), for films grown with $\mathrm{P}_{\mathrm{O} 2}$ of 0.20 mbar and 0.15 mbar, respectively. Colors are in log scale. Close-up of the $\mathrm{BaTiO}_{3}$ $(204)_{p c}$ peak showing no domains (g) or domains (h), corresponding to the red squares in (e) and (f) respectively.

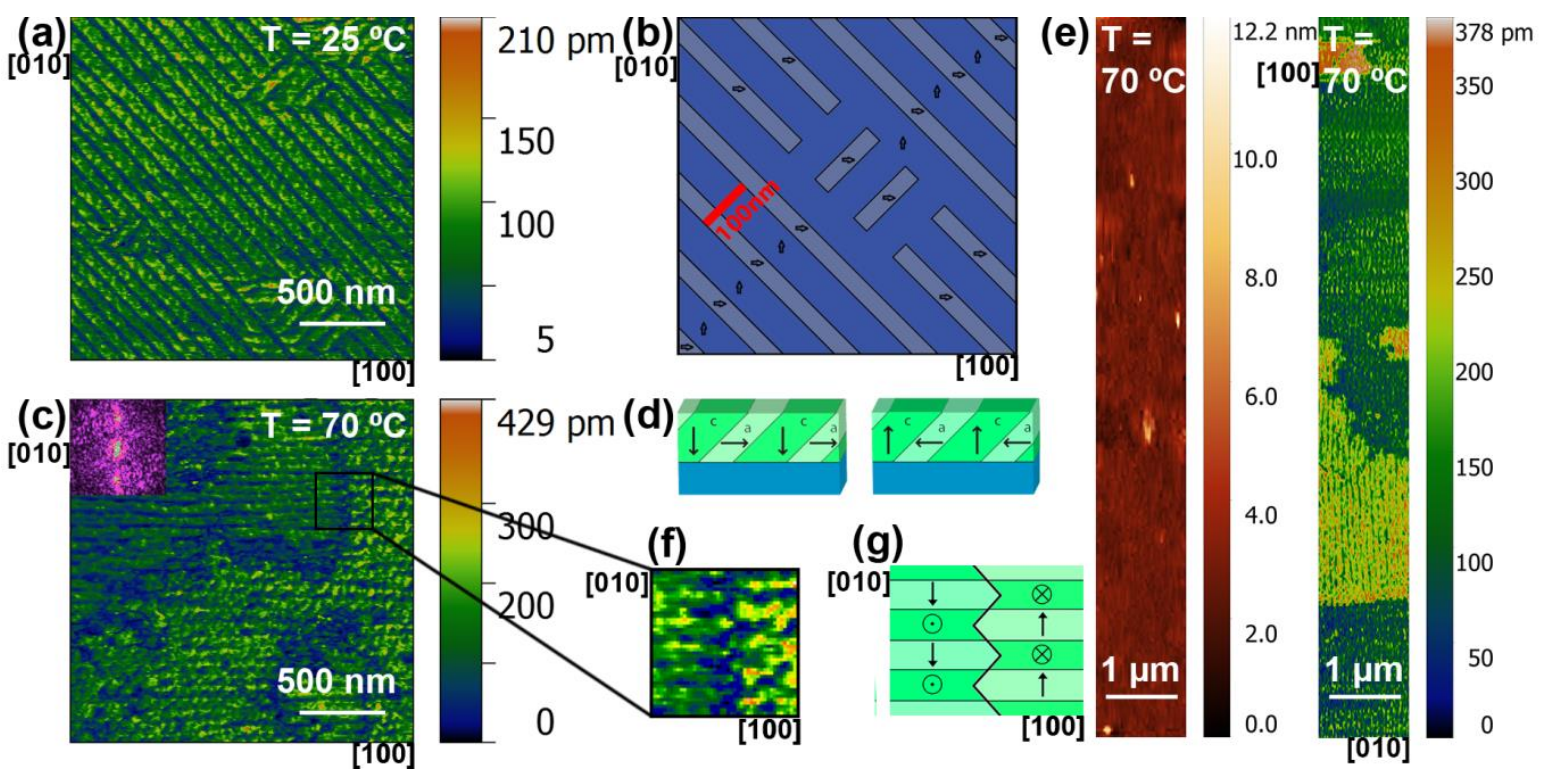

Figure 3. a) Lateral amplitude PFM image at room temperature. b) Model for the ferroelectric polarization in the $\mathrm{a}_{1} / \mathrm{a}_{2}$ domains as a top view. c) Lateral amplitude PFM image at $70{ }^{\circ} \mathrm{C}$. Inset: Fast Fourier Transform, the clear periodicity peaks correspond to the domain periodicity. d) $3 \mathrm{D}$ model for the ferroelectric polarization in these two sets of a/c domains forming superdomains. e) Topography and lateral amplitude PFM images at $70{ }^{\circ} \mathrm{C}(10 \mu \mathrm{m}$ 


\section{WILEY-VCH}

image length), without disturbance of the nanodomain structures, except for the superdomains. f) Some superdomain boundaries produce zig-zag domains. g) Model of the polarization in the zig-zag domains.
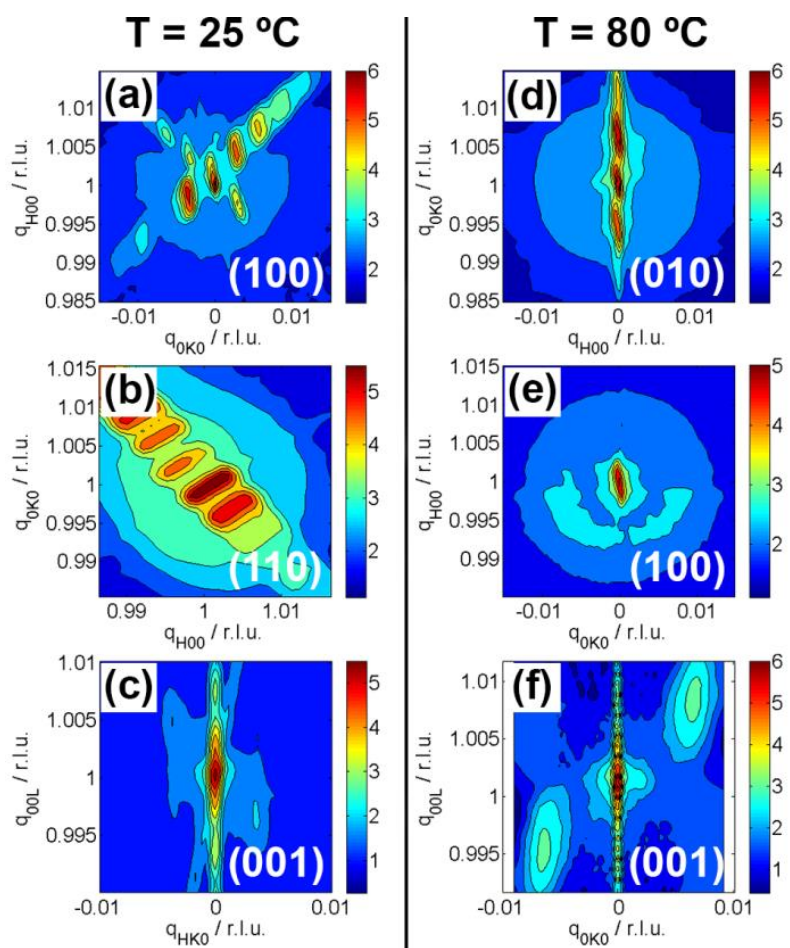

Figure 4. RSM around (100) (a), (110) (b) and (001) (c) Bragg peaks at room temperature; RSM around (010) (d), (100) (e) and (001) (f) Bragg peaks at $80^{\circ} \mathrm{C}$. Colors are in $\log (10)$ scale.
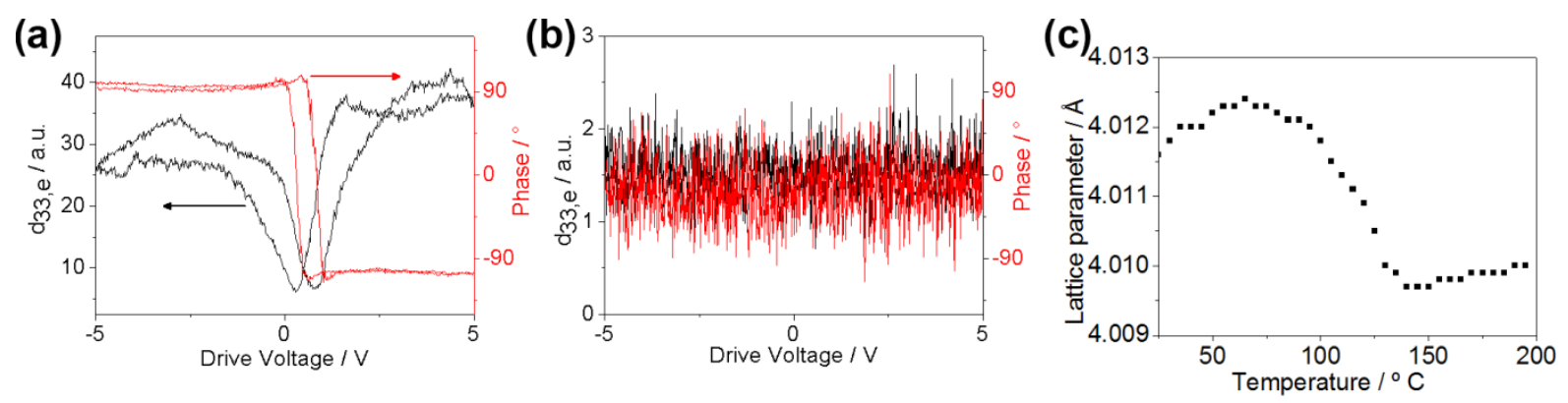

Figure 5. PFM hysteresis loop of an effective piezoelectric constant $\mathrm{d}_{33, \mathrm{e}}$ and the corresponding phase at a) $70{ }^{\circ} \mathrm{C}$ (lower than $\mathrm{T}_{\mathrm{C}}$ ), and at b) $140{ }^{\circ} \mathrm{C}$ (higher than $\mathrm{T}_{\mathrm{C}}$ ). c) $\mathrm{BaTiO}_{3}$ out-of-plane lattice parameter obtained from a $\theta-2 \theta$ scan around (001) Bragg peak versus temperature. 


\section{WILEY-VCH}

\section{$\mathrm{BaTiO}_{3}$ thin films have been grown by Pulsed Laser Deposition on low-strain $\mathrm{NdScO}_{3}$}

substrates. A room-temperature monoclinic $\mathrm{ca}_{1} / \mathrm{ca}_{2}$ periodic domain configuration with inplane and additional symmetry-lowering out-of-plane polarization is stabilized; while above $50{ }^{\circ} \mathrm{C}$, an a/c domain configuration with very long domain walls is observed. This rich phase diagram with domains and lowered symmetry is promising for high piezoelectric response.

$\mathrm{BaTiO}_{3}$

Arnoud S. Everhardt*, Sylvia Matzen, Neus Domingo, Gustau Catalan, Beatriz Noheda*

Ferroelectric domain structures in low-strain $\mathrm{BaTiO}_{3}$

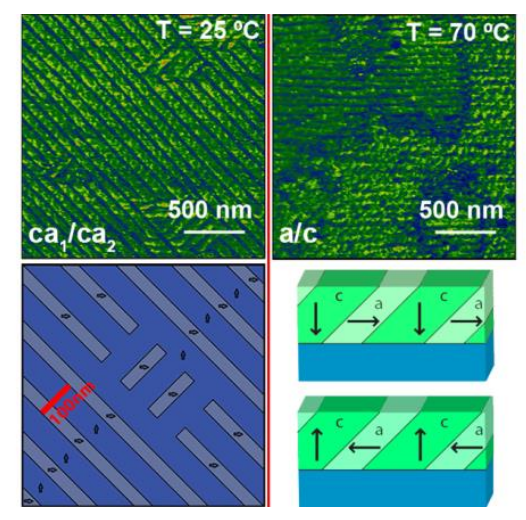


Copyright WILEY-VCH Verlag GmbH \& Co. KGaA, 69469 Weinheim, Germany, 2013.

\title{
Supporting Information
}

\author{
Ferroelectric domain structures in low-strain $\mathrm{BaTiO}_{3}$
}

Arnoud S. Everhardt*, Sylvia Matzen, Neus Domingo, Gustau Catalan, Beatriz Noheda*

(a)

[010]D.

(d)

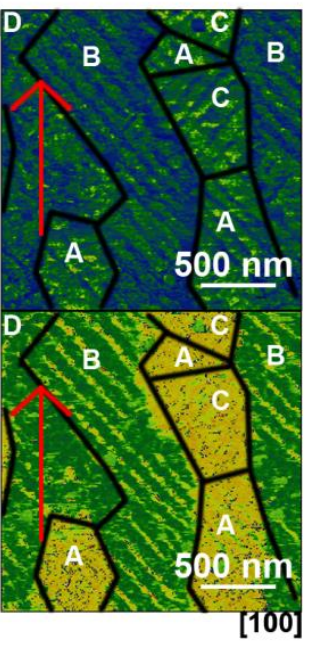

(b)

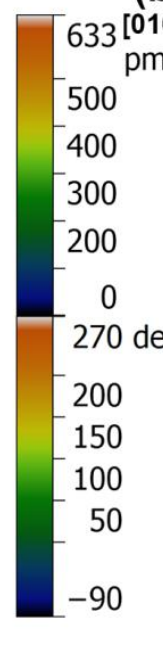

(b)

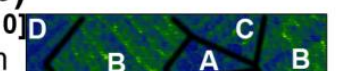

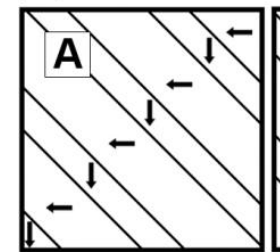
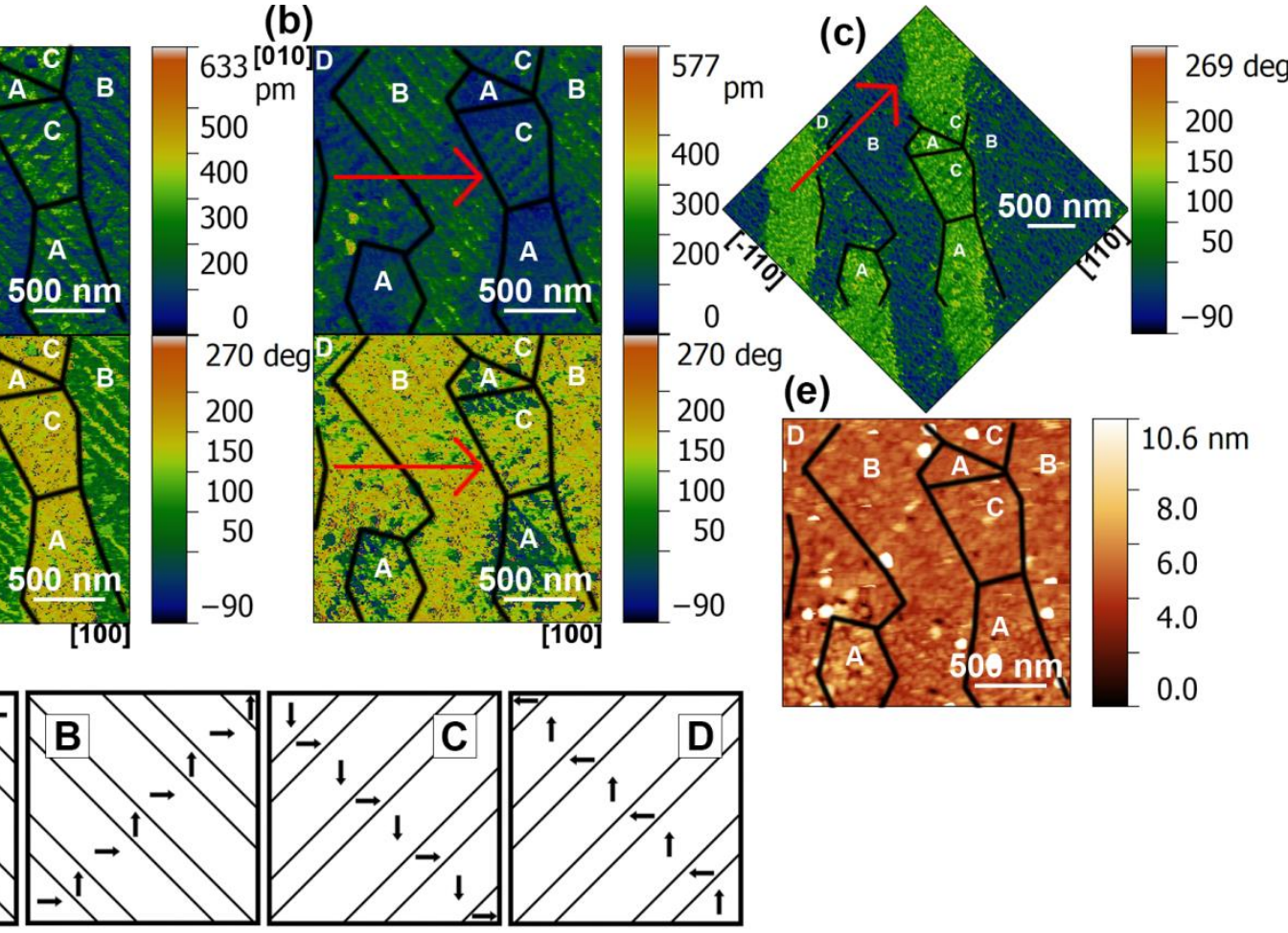

Figure S1. Lateral amplitude and phase PFM images taken of a single area at room temperature. The polarization sensitivity for the lateral PFM image is indicated by the red arrow, vertical (a), horizontal (b) and diagonal (c). d) Model of the four types of superdomains found in these images, labelled A, B, C and D as in Figures a), b) and c). An example of how these domains can be reconstructed can be obtained by looking at superdomains A and B in (a) and (b). In (a), superdomain A has high phase for the $[0-10]_{p c}$ oriented polarization domains and intermediate phase for the $\langle 100\rangle_{p c}$ oriented polarization domains. Then in superdomain $\mathrm{B}$, the same intermediate phase can be found for the $\langle 100\rangle_{p c}$ oriented polarization domains and high phase for the $[010]_{p c}$ oriented polarization domains. In (b), superdomain A has low ([-100] $]_{p c}$ oriented polarization) and intermediate $\left(\langle 010\rangle_{p c}\right.$ oriented polarization) phases for its domains, and superdomains B has high ([100 $]_{p c}$ oriented polarization) and intermediate $\left(\langle 010\rangle_{p c}\right.$ oriented polarization) phases for its domains. Similar arguments can be made for the other superdomains and the sample oriented at the $[110]_{p c}$ axis. e) Topography image of the area investigated by PFM. The superdomain walls are often correlated to topographic defects, suggesting that those defects are domain wall nucleation points. 


\section{WILEY-VCH}

(a)

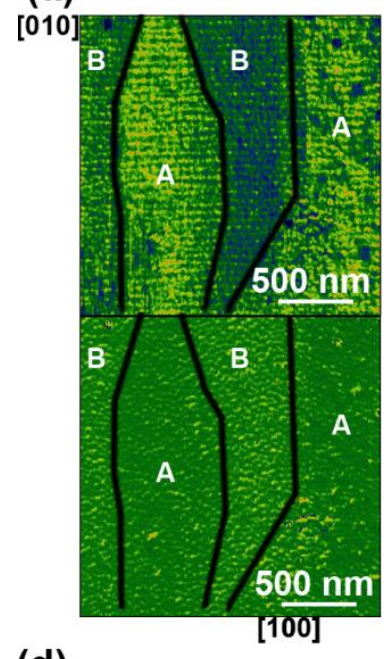

(d)

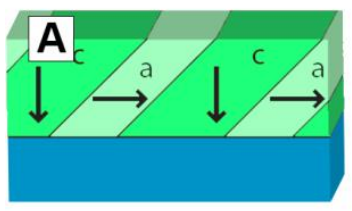

(b)

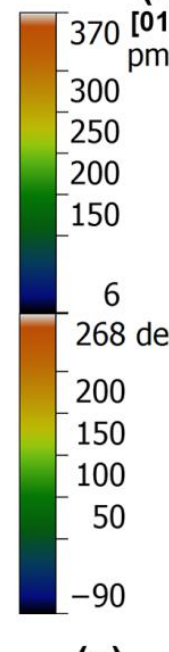

(e)

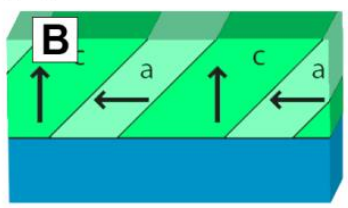

(c)

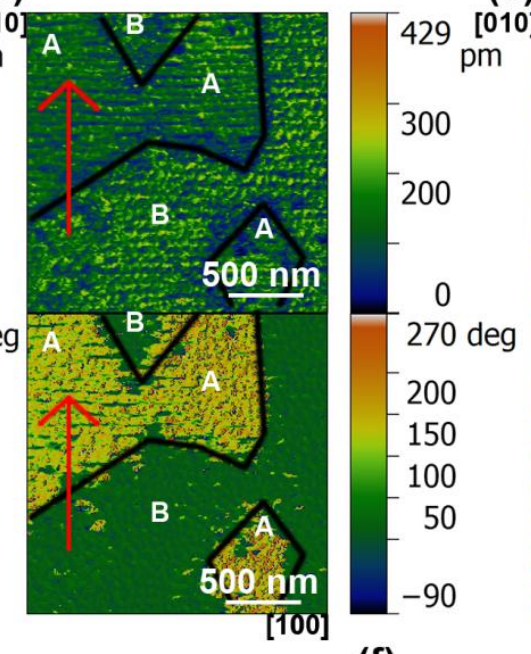

(f)

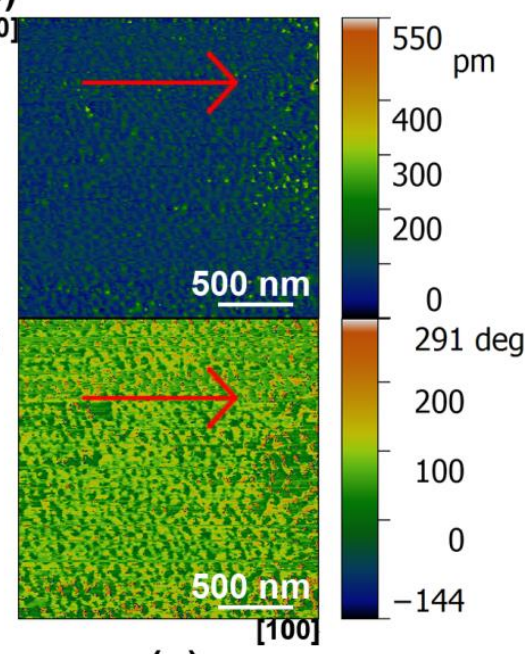

(g)

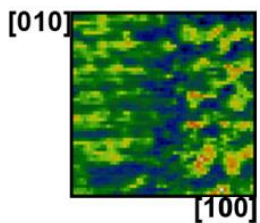

Figure S2. PFM images taken at $70{ }^{\circ} \mathrm{C}$. Vertical PFM image (a) and lateral PFM image with the polarization sensitivity along $[010]_{p c}$ (b) show domain contrast, while the lateral PFM image with polarization sensitivity along $[100]_{p c}$ (c) doesn't show any domain contrast. So these PFM results prove that the in-plane polarization of $\mathrm{BaTiO}_{3}$ thin films is only along the $[010]_{p c}$ direction. Superdomains A (d) and B (e) are observed, where some of the superdomain walls (f) form zig-zag walls (g). The domain configuration can be found by considering the amplitudes of the piezoresponse. High piezoresponse (in-plane domains) of superdomain B crosses into intermediate piezoresponse (out-of-plane domains) of superdomain A; and intermediate piezoresponse (out-of-plane domains) of B crosses into low piezoresponse (in-plane domain rotated $180^{\circ}$ ) of A. Similar observations can be made for the Vertical PFM image.

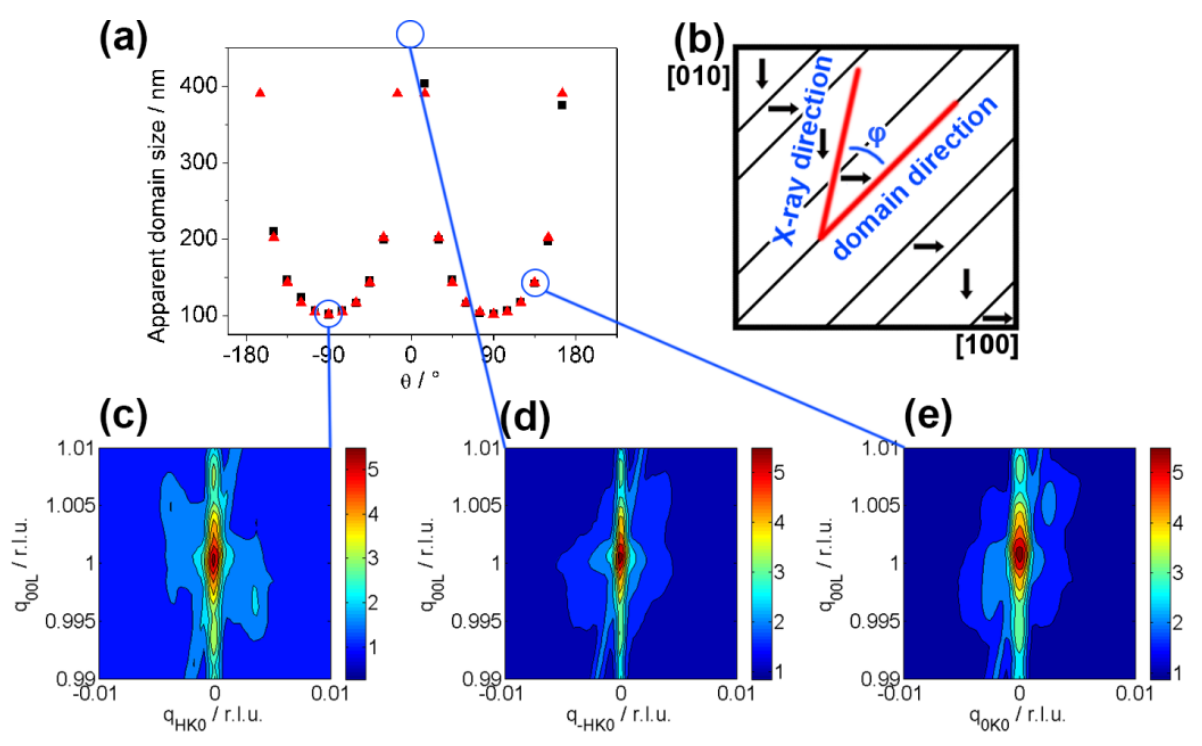

Figure S3. a) Measured (black squares) and calculated (red triangles) apparent domain size found from the intensity modulation in the diffuse scattering around the (001) Bragg peak, for 


\section{WILEY-VCH}

different azimuthal angles of rotation $(\varphi)$ around the $[001]_{p c}$ axis. b) Model for the calculation of apparent domain size. Domains have periodicity along $[110]_{p c}$, and the in-plane axis measured by X-rays in the RSM around the $(001)_{p c}$ Bragg peak is determined by the angle $\varphi$. Then the apparent domain size is a function of the sine of that angle: apparent domain size = domain size / $\sin (\varphi)$. In the figure it can be seen that the length that X-ray direction vector takes to cross one domain is equal to the apparent domain length observed in the RSM. The graph can be illustrated by RSMs: the in-plane spacing is maximum for a $\varphi$ of $-90^{\circ}$ (c), corresponding to a minimum domain size; the domain peaks are absent for a $\varphi$ of $0^{\circ}$ (d), corresponding to infinite domain size; and the domain peaks are in between both situations for a $\varphi$ of $135^{\circ}$ (e).
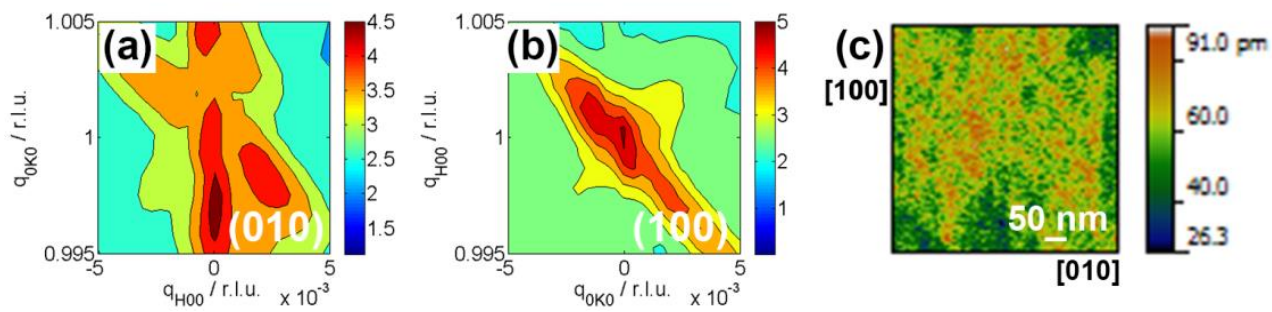

Figure S4. XRD RSM around the $(010)_{p c}$ (a) and (100) $)_{p c}$ (b) Bragg peaks at $\mathrm{T}=30^{\circ} \mathrm{C}$ after fast cooldown. In both RSMs intensity modulation in the diffuse scattering along $[110]_{p c}$ can be observed, corresponding to the $\mathrm{ca}_{1} / \mathrm{ca}_{2}$ phase. On top of that, the $(010)_{p c}$ Bragg peak RSM gives intensity modulation along [010] $]_{p c}$, while no such modulation is observed around the $(100)_{p c}$ Bragg peak, which is the signature of the a/c phase of these $\mathrm{BaTiO}_{3}$ thin films. So it can be concluded that both phases coexist simultaneously in this fast cooled down film. (c) Amplitude out-of-plane PFM image of a small area showing indication of both $\mathrm{ca}_{1} / \mathrm{ca}_{2}$ and a/c phases. The observation of $\mathrm{ca}_{1} / \mathrm{ca}_{2}$ domains walls along [110] in the out-of-plane mode could be either due to the real out-of-plane polarization or cross-talking with the in-plane components (as discussed previously). In addition, the domains with walls along the [100] direction, consistent with those of the a/c domains observed at high temperature are weakly visible. 